\title{
Clinical Application of the Cardio-Ankle Vascular Index in Asymptomatic Healthy Koreans
}

\author{
Su-Yeon Choi \\ Division of Cardiology, Department of Internal Medicine, Seoul National University Hospital, \\ Healthcare System Gangnam Center, Seoul National University College of Medicine, Seoul, \\ Republic of Korea
}

\section{Key Words}

Arterial stiffness · Coronary artery disease $\cdot$ Fat burden $\cdot$ Koreans $\cdot$ Risk factor

\begin{abstract}
Background: Arterial stiffness has been established as a surrogate marker for the prognosis of cardiovascular disease. Arterial stiffness is also a predictor of future cardiovascular events, and is the earliest detectable manifestation of adverse structural and functional changes to blood vessel walls. Summary and Key Messages: The cardio-ankle vascular index (CAVI) is an index representing the stiffness of the entire arterial segments from the aorta to the ankle independent of the blood pressure at the time of the measurement. This paper provides an overview of the clinical application of arterial stiffness measurement by CAVI in asymptomatic Koreans. It includes the association between cardiometabolic risk factors and CAVI, and the relation between CAVI and asymptomatic coronary artery disease.
\end{abstract}

(c) 2016 S. Karger AG, Basel

\section{Introduction}

Arterial stiffness, evaluated by various methods such as pulse wave velocity (PWV), augmentation index (AIx) and beta stiffness index, has been established as a marker of cardiovascular disease (CVD) and a risk factor in the progression of atherosclerosis [1]. However, most of these parameters were affected by the blood pressure at the time of measurement. The cardio-ankle vascular index (CAVI) reflects the stiffness of the whole arterial segments including the aorta, femoral artery and tibial artery [2] and is not affected by blood pressure during the measurement. 
Fig. 1. CAVI scores by age between males and females among CVD risk-free Koreans. CAVI = $5.0+0.048 \times$ age (year) in male $\left(r=0.629, r^{2}=0.395, \mathrm{p}<0.001\right)$; CAVI $=4.8+0.045 \times$ age (year) in female $\left(r=0.671, r^{2}=0.450, \mathrm{p}<\right.$ $0.001)$ by simple linear regression. Modified from Choi et al. [3].
Choi et al.: Clinical Application of the Cardio-Ankle Vascular Index in Asymptomatic Healthy Koreans

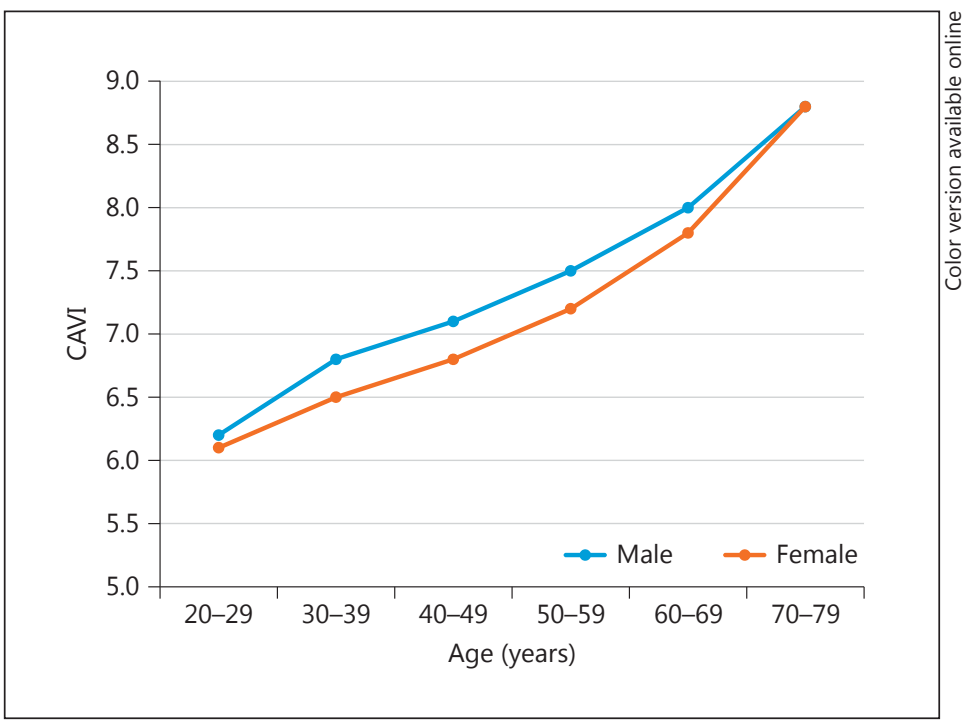

This paper provides an overview of the clinical application of the CAVI to evaluate arterial stiffness in an asymptomatic Korean population.

\section{Age- and Gender-Stratified Value of the CAVI in Healthy Koreans}

We reported the age- and gender-stratified value of the CAVI in healthy Koreans to evaluate the effect of age on arterial stiffness [3]. Healthy and normotensive subjects aged 20-79 years had no history of CVD and were free from taking medications for hypertension, diabetes mellitus or dyslipidemia ( $\mathrm{n}=1,380 ; 44.1 \%$ males). The CAVI increased linearly with age (fig. 1), whereas systolic blood pressure, diastolic blood pressure and pulse pressure did not. The CAVI in men was higher than that in women among subjects aged 30-59 years. The differences between the genders were not significant in the subjects over 60 years of age.

These data suggest that age is the dominant risk factor for the progression of arterial stiffness, and that the CAVI might be a sensitive marker of the arterial aging process above and beyond the conventional upper-arm blood pressure.

In the Anglo-Cardiff Collaborative Trial with a large cohort of healthy, normotensive individuals [4], aortic PWV correlated significantly with age. In contrast to CAVI, age-related changes in aortic PWV and AIx did not appear linear, with AIx increasing more in younger individuals, whereas the changes in aortic PWV were more prominent in older individuals over 50 years of age.

The age- and sex-specific CAVI in healthy Koreans may play a role as the baseline value to understand the effect of CVD risk factors on arterial stiffness and to evaluate the effect of treatment of CVD risk factors in younger and older populations.

\section{The Association of Arterial Stiffness with Different Fat Compartments}

We investigated the association of fat burden with CAVI in a symptomatic population ( $n=260$ ) [5]. Visceral adipose tissue, epicardial adipose tissue (EAT) and the subcutaneous to visceral adipose tissue ratio have shown significant correlations with arterial stiffness 


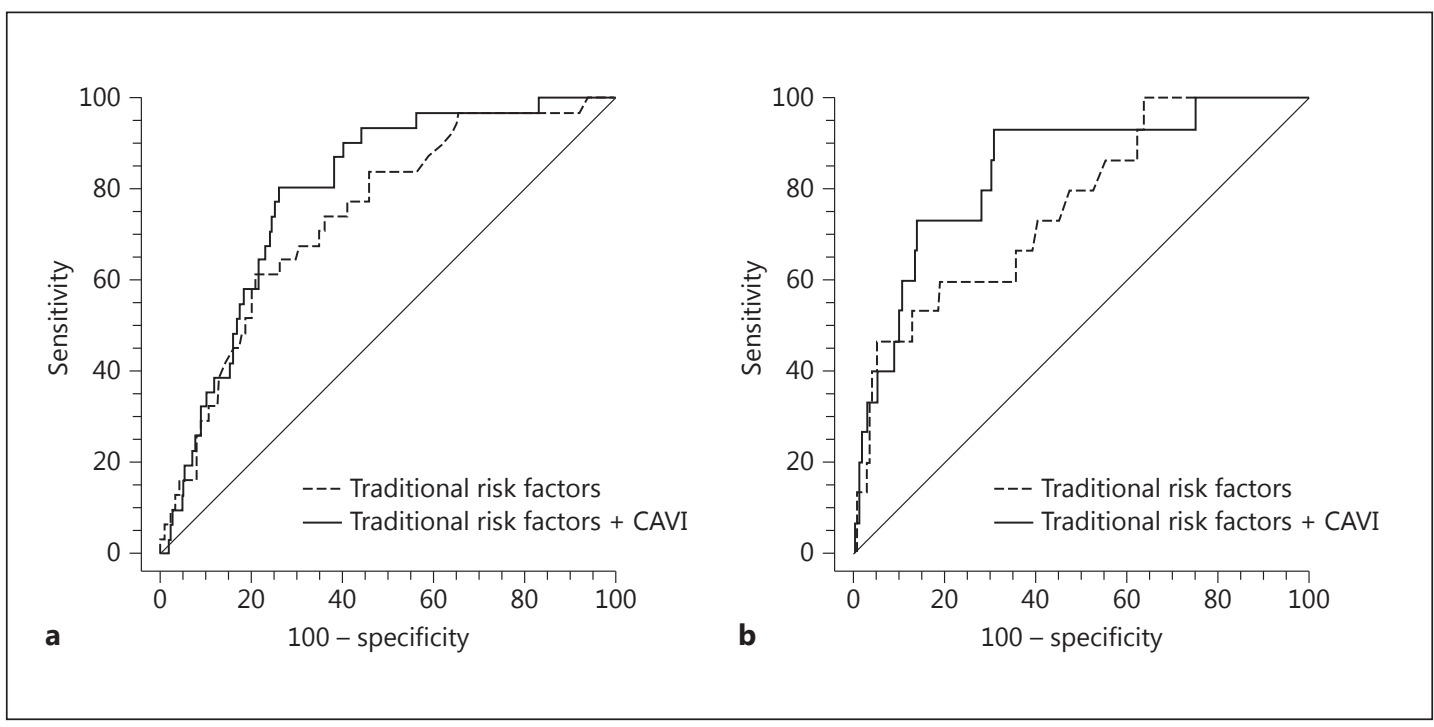

Fig. 2. Comparison of the area under the curves for predicting CAC $\geq 300$ (a) and coronary stenosis $\geq 50 \%$ (b) for CAVI in addition to traditional risk factors including age $\geq 50$ years, gender, hypertension, diabetes mellitus, dyslipidemia, and high-sensitive C-reactive protein. The area under the curves increased from 0.739 to 0.791 ( $p=0.023$ ) (a) and from 0.761 to $0.842(p=0.032$ ) (b). Modified from Park et al. [7].

measured by CAVI. EAT showed an independent association with arterial stiffness by multivariable correlation analysis. However, there was no significant difference in CAVI among body mass index categories in both genders.

Different fat compartments have shown different correlations with CAVI. Visceral obesity, rather than general obesity per se, is a possible risk factor for increased arterial stiffness. EAT showed the strongest correlation with CAVI, which may be due to greater metabolic activity of EAT.

\section{Nonalcoholic Fatty Liver Disease as a Risk Factor for Increased Arterial Stiffness}

Nonalcoholic fatty liver disease (NAFLD) is associated with risk factors for CVD. We investigated whether NAFLD is associated with arterial stiffness in apparently healthy subjects without any known liver diseases $(n=2,954)$ [6]. NAFLD diagnosed via typical ultrasonography was found in $42.3 \%$. When adjusted for age, sex, body mass index, waist circumference, smoking status, diabetes, and hypertension, NAFLD was associated with a $32 \%$ increase in the risk of increased arterial stiffness (age- and sex-specific highest quartile of the CAVI). The risk of arterial stiffness increased according to the severity of NAFLD [adjusted odds ratio (95\% confidence interval), 1.20 (0.96-1.50) vs. 1.59 (1.21-2.08), for mild vs. moderate-to-severe, respectively]. In subgroup analysis, the association between NAFLD and arterial stiffness was significant in the younger and nonobese groups. The presence of cardiometabolic risk factors in the older and obese groups may attenuate the prediction.

These results suggest that NAFLD is a risk factor for increased arterial stiffness, and CAVI is a useful tool to evaluate the risk, especially in the younger and nonobese groups. 


\section{Relation between Arterial Stiffness and Coronary Artery Calcification or Stenosis}

We investigated the association of CAVI with the severity of coronary artery calcification (CAC) and coronary stenosis by coronary computed tomography angiography in an asymptomatic population without coronary artery disease $(n=549)$ [7]. After adjustment for potential confounders, including age, gender, hypertension, diabetes mellitus, and dyslipidemia, a predefined cutoff value of CAVI $\geq 8$ was associated with advanced CAC (CAC $\geq 300$ ) and significant coronary stenosis (stenosis $\geq 50 \%$ ). Specifically, the adjusted odds ratio ( $95 \%$ confidence interval) of CAC $\geq 300$ and coronary stenosis $\geq 50 \%$ was 3.57 (1.92-6.66) and 2.81 (1.13-7.00), respectively. Additional inclusion of CAVI improved the predictive power of the receiver operating characteristic curves for predicting coronary atherosclerosis based on traditional risk factors (fig. 2). CAVI reflects coronary atherosclerosis and may be used as a screening tool for assessing subclinical atherosclerotic burden in an asymptomatic population.

\section{Conclusions}

In Korea, arterial stiffness measured with CAVI was reported to be related to cardiometabolic risk factors such as EAT and NAFLD. Therefore, EAT and NAFLD were independent risk factors for increased arterial stiffness in an asymptomatic healthy population. CAVI was associated with advanced CAC and significant coronary stenosis. Arterial stiffness measurement by CAVI may also play potential roles in reflecting coronary atherosclerosis. In order to explain these mechanisms, further studies are needed to evaluate the association between CAVI and various biochemical materials which cause metabolic disorders and coronary artery disease.

\section{Disclosure Statement}

There are no conflicts of interest.

\section{References}

1 Laurent S, Cockcroft J, Van Bortel L, Boutouyrie P, Giannattasio C, Hayoz D, Pannier B, Vlachopoulos C, Wilkinson I, Struijker-Boudier H: Expert consensus document on arterial stiffness: methodological issues and clinical applications. Eur Heart J 2006;27:2588-2605.

2 Shirai K, Hiruta N, Song M, Kurosu T, Suzuki J, Tomaru T, Miyashita Y, Saiki A, Takahashi M, Suzuki K, Takata M: Cardio-ankle vascular index (CAVI) as a novel indicator of arterial stiffness: theory, evidence and perspectives. J Atheroscler Thromb 2011;18:924-938.

3 Choi SY, Oh BH, Bae Park J, Choi DJ, Rhee MY, Park S: Age-associated increase in arterial stiffness measured according to the cardio-ankle vascular index without blood pressure changes in healthy adults. J Atheroscler Thromb 2013;20:911-923.

4 McEniery CM, Yasmin, Hall IR, Qasem A, Wilkinson IB, Cockcroft JR: Normal vascular aging: differential effects on wave reflection and aortic pulse wave velocity: the Anglo-Cardiff Collaborative Trial (ACCT). J Am Coll Cardiol 2005;46:1753-1760.

5 Park HE, Choi SY, Kim HS, Kim MK, Cho SH, Oh BH: Epicardial fat reflects arterial stiffness: assessment using 256-slice multidetector coronary computed tomography and cardio-ankle vascular index. J Atheroscler Thromb 2012;19:570-576.

6 Chung GE, Choi SY, Kim D, Kwak MS, Park HE, Kim MK, Yim JY: Nonalcoholic fatty liver disease as a risk factor of arterial stiffness measured by the cardioankle vascular index. Medicine (Baltimore) 2015;94:e654.

7 Park JB, Park HE, Choi SY, Kim MK, Oh BH: Relation between cardio-ankle vascular index and coronary artery calcification or stenosis in asymptomatic subjects. J Atheroscler Thromb 2013;20:557-567. 